\title{
The effect of intermittent renewables on the electricity price variance*
}

\author{
David Wozabal ${ }^{\dagger} \quad$ Christoph Graf $f^{\ddagger}$ David Hirschmann ${ }^{\S}$
}

28.12.2014

\begin{abstract}
The dominating view in the literature is that renewable electricity production increases the price variance on spot markets for electricity. In this paper, we critically review this hypothesis. Using a static market model, we identify the variance of the infeed from intermittent electricity sources (IES) and the shape of the industry supply curve as two pivotal factors influencing the electricity price variance. The model predicts that the overall effect of IES infeed depends on the produced amount: while small to moderate quantities of IES tend to decrease the price variance, large quantities have the opposite effect. In the second part of the paper, we test these predictions using data from Germany, where investments in IES have been massive in the recent years. The results of this econometric analysis largely conform to the predictions from the theoretical model. Our findings suggest that subsidy schemes for IES capacities should be complemented by policy measures supporting variance absorbing technologies such as smart-grids, energy storage, or grid interconnections to ensure the build-up of sufficient capacities in time.
\end{abstract}

Keywords: Electricity Spot Markets; Photovoltaics; Wind Power; EPEX; Merit Order

\section{Introduction}

In the last decades electricity markets were liberalized in many countries, most of which established electricity exchanges where electricity is traded on an hourly basis. Since the possibilities of storing electricity are limited and demand is highly inelastic, electricity prices exhibit strong daily, weekly, and yearly seasonalities which are mainly driven by fluctuating demand. This peculiar property of electricity is shared by no other commodity and prompted active research investigating the competitiveness of the markets, the statistical properties of the price process, and the determinants of price formation.

\footnotetext{
*We want to express our thanks to Klaus Gugler, Felix Höffler, Franz Wirl, and the participants of the Mannheim Energy Conference 2013 for stimulating discussions and valuable inputs on a draft version of this paper. Additionally, the paper greatly benefited from the comments and suggestions of two anonymous referees. David Hirschmann was partially supported by the WWTF (Project Number: MA09-019).

${ }^{\dagger}$ Technical University Munich, TUM School of Management, Arcisstrasse 21, 80333 Munich, Germany. Tel.: +49-89-289-25043, Fax: +49-89-289-25488, david.wozabal@tum.de

$\ddagger$ Florence School of Regulation - Climate. European University Institute, Via delle Fontanelle 19, 50014 San Domenico di Fiesole, Italy, and Faculty of Business, Economics, and Statistics. University of Vienna, Oskar-Morgenstern Platz 1, 1090 Vienna, Austria.

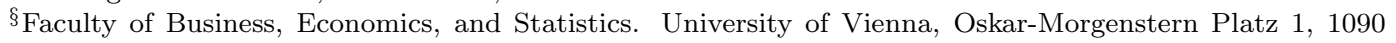
Vienna, Austria.
} 
In the last years the debate centered on the transformation of electricity production either driven by the goal to decrease the carbon footprint of the industry, or by the phasing out of nuclear energy as, e.g., in Germany. In either case, the build-up of renewable energy sources is expected to play an important role in reaching these goals. For the understanding of the dynamics of electricity prices, the most interesting aspect of this transition is the interplay of large intermittent production capacities with the above mentioned characteristics of electricity demand and insufficient storage capacities.

The impact of intermittent electricity sources (IES) on electricity prices is investigated in many papers. Most of them focus on the price level and it is widely agreed on that in the short and medium run an increase in IES reduces the average spot prices (see, e.g., Green and Vasilakos, 2010; Jacobsen and Zvingilaite, 2010; Woo et al, 2011; Paraschiv et al, 2014). ${ }^{1}$ In this study, we focus on another aspect: we assess the impact of intermittent production on the variance of electricity prices.

The price variance is an important quantity for several reasons. Its evolution is a major determinant when investing in plants, for which the return is directly connected to the shortterm variation of prices such as electricity storage plants. These plants earn money by buying electricity when prices are low and selling it when prices are high. The higher the price variance, and therefore the difference between high and low prices, the more profitable this strategy is. Hence, high price variance directly translates to higher earnings of storages and therefore higher incentives to invest in this type of plants (see Muche, 2009; Dunne and Mu, 2010). Likewise the returns on investments in smart grid infrastructure depend heavily on the variance of prices. The higher the price variance, the higher the incentive to invest in smart grid and metering technology that smoothens the demand. Since these technologies are necessary to deal with the challenges induced by an increased share of IES production, understanding the mechanisms that drive price variance on electricity exchanges is of importance to academics, policy makers, and investors alike.

The extant literature is not clear on the issue of price variance for two reasons. First, many studies refer to price variability, a vague term for which many possible definitions are used, e.g., the range of observable prices, the frequency of price spikes, the price variance, or the volatility. Second, findings are contradictory. Most authors predict an increase in price variability due to IES. However, some empirical works find a reduced price variability of power prices as the amount of intermittent production increases. In this paper, we introduce a theoretical framework that reconciles these seemingly contradictory findings and is consistent with the data observed on the German electricity market.

As mentioned above, different conclusions concerning the evolution of price variability have been drawn in literature so far. Among the theoretical works predicting an increased price variability is Milstein and Tishler (2011) who consider a two stage game for electricity producers. In the first stage, energy producers decide which share of their production portfolio will be intermittent. In the second stage, energy is produced accordingly, but with some probability the IES cannot be exploited which causes price spikes due to inelastic demand. This implies that build-up IES can increase price volatility. Green and Vasilakos (2010) simulate the impact of wind generation on the spot market prices in the UK for the year 2020. They rely on real world wind data, information on existing and planned wind farms, and demand data. Assuming a competitive market, they simulate monthly price distributions. The results imply that volatility of prices increases in future and that the magnitude of the increase depends not only on the variation of wind output but also on the competitiveness of the market. In Chao (2011)

\footnotetext{
${ }^{1}$ Note that in the long run the price reducing effect could be offset by adjustments in the conventional capacities (see, e.g., de Miera et al, 2008).
} 
a central planer chooses a production portfolio and sets prices as to maximize expected social welfare. Stochastic demand depends on prices, and supply is subject to random outages or intermittence. For two different pricing schemes, scenarios with and without IES are compared. While average prices decrease due to IES, the load-weighted standard deviations increase.

Other papers focus on historical data and find that price variance has increased due to IES. Woo et al (2011) examine the effect of wind generation on spot prices in Texas by means of a (partial-adjusted) linear regression model. By extrapolation of their models they argue that price variance tends to increase if wind generation is raised. More recently, Ketterer (2012) analyzed the German electricity market by using a GARCH model. She finds that an increased production from wind reduces the mean price but increases the variance while an increased load shows the opposite effects.

The conclusions concerning price variability are ambiguous in Jónsson et al (2010) who study the effects of wind energy forecasts on prices in Denmark. They consider different levels of wind penetration, that is, the percentage of electricity produced by wind turbines. Their findings suggest that higher predicted wind penetration lowers the mean spot price and reduces the intra-day variance of the prices. Still, they conclude that price volatility will increase in future, due to the varying price patterns induced by different wind penetration scenarios. Jacobsen and Zvingilaite (2010) also study the current market situation in Denmark on a broad level and focus on the large share of IES. With respect to prices, they conclude that prices fluctuate more but that the frequency of peak prices is reduced.

Tveten et al (2013) report that photovoltaic (PV) infeeds in Germany have decreased price variance substantially from 2009-2011. They compare days with similar electricity demand and different levels of PV production and find that on days with high PV infeeds the price variance is lower. This is attributed to the merit order effect: under high PV power penetration, the production mix is changed, i.e., peak power plants with high short-run marginal costs are used less. Therefore, prices stay at a lower level and do not jump up to peak-load prices, which reduces fluctuations.

The merit order effect also forms the base of this paper, but we study it more systematically and in a broader context for wind and PV energy. We contribute to the discussion by showing that IES can either increase or decrease price variance. For our theoretical investigations, we use a static market model with random residual demand influenced by IES infeed. In this setup, we investigate how variance changes, ceteris paribus, as the IES capacity grows. We identify two factors driving the price variance: (1) the curvature of the supply function (merit order), and (2) the distribution of the random IES supply. To test our theoretical findings, we perform statistical analysis on daily price variances from Germany and are able to verify the existence of the two effects. Furthermore, our statistical analysis provides evidence that the merit order in the German market is inverse S-shaped.

Our choice of the variance as a measure of dispersion of electricity prices is motivated by its nice theoretical properties: both the market model as well as the decomposition of the price variance into its components in the empirical part of the paper hinge on that choice. Other measures of variation such as the mean absolute deviation or the standard deviation do not permit a similar analysis. We therefore choose the variance over its alternatives, although it presents us with certain challenges in the econometric analysis due to its sensitivity to outliers.

The remainder of this paper is structured as follows. In Section 2, we detail the theoretical approach, identifying the effects which influence the price variance. In Section 3, we analyze the effects of increased intermittent electricity production on the variance of electricity prices in an econometric model. Section 4 is devoted to the policy implications of our findings, while Section 5 concludes the paper. 


\section{Theory}

In the following, we provide a theoretical framework explaining how IES infeeds affect the price variance. We first explain the market model and then give an intuitive idea of two opposing effects that intermittent production has on the distribution of the electricity prices. We conclude with a formal analysis, which identifies the relevant factors influencing the price and show that an increased IES infeed does not necessarily increase the price variance, but may even lower it.

Our investigations are based on a standard static market model with inelastic, random aggregate demand for electricity $Q$. As common in the literature, we start with the assumption of a competitive convex market supply curve $S$, which equals the aggregate industry cost function (see e.g., Barlow, 2002; Kim and Knittel, 2006; Redl et al, 2009; Arocena et al, 2012). The assumption of convexity will be relaxed later on to implicitly incorporate dynamic aspects of the plant dispatch problem of electricity producers. The market price is obtained as $S(Q)$, i.e., by intersecting the inelastic demand with the supply curve. We therefore implicitly assume that no player is able to exercise market power.

In the setting outlined above, the most natural way to include intermittent production would be to change the supply function. However, for the purpose of our model, we find it more convenient to subtract the intermittent production $I$ from the demand. In this way we obtain the so called residual demand $Q-I$, which is the demand that has to be covered by conventional plants. The obvious advantage of this kind of modeling is that all the stochastic quantities, i.e., the demand and the intermittent infeed, enter the residual demand while the supply curve remains deterministic. Incorporating IES via a change in the supply function or via the residual demand is equivalent, if intermittent production is always fed-in regardless of the price, i.e., if it merely shifts the demand curve to the left. Indeed, typically intermittent sources of electricity have marginal costs close to zero and therefore supply all the energy they produce into the grid as long as prices are positive. Additionally, many countries subsidize intermittent renewable production by guaranteed feed-in tariffs which also include preferential access to the market. Therefore it is quite standard to model renewable production via residual demand (see e.g., Graf and Wozabal, 2013; Hirth, 2013; Wagner, 2014). ${ }^{2}$

Figure 1 intuitively explains how an increase in IES infeed affects the market price in our model. The $x$-axis indicates the quantity of electricity and the $y$-axis the price. The densities on the $x$-axis represent the stochastically varying residual demands $X=Q-I$, which are mapped to the stochastic prices $P=S(X)$ whose densities are drawn over the $y$-axis. In Figure 1(a), the increased amount of IES shifts the residual demand to the left to a region where the supply curve is flatter. If the shape of the residual demand distribution remains unchanged, the variance of the prices decreases. If, however, an increased share of intermittent production changes the shape of the distribution of $X$, then the situation is ambiguous. For example, if the density of the residual demands broadens by an increase in intermittent production, then the variance could increase, even though the residual demand is pushed into the flat area of the supply function as is illustrated in Figure 1(b). Hence, the overall effect of intermittent production depends crucially on the shape of the distribution of the residual demand and on the slope of the supply function. These considerations imply that the build-up of IES can either increase or decrease price variance, depending on which effect has a stronger influence.

We state this in a more rigorous way: Let the supply function $S: \mathbb{R} \rightarrow \mathbb{R}$ be increasing,

\footnotetext{
${ }^{2}$ If there are no feed-in tariffs, the providers of IES would have an incentive to switch off their production in case the price falls below zero. Hence, IES infeed would depend on market prices. For the sake of simplicity and because the current set of assumptions fits market conditions in most markets, we abstain from this complication of the model.
} 


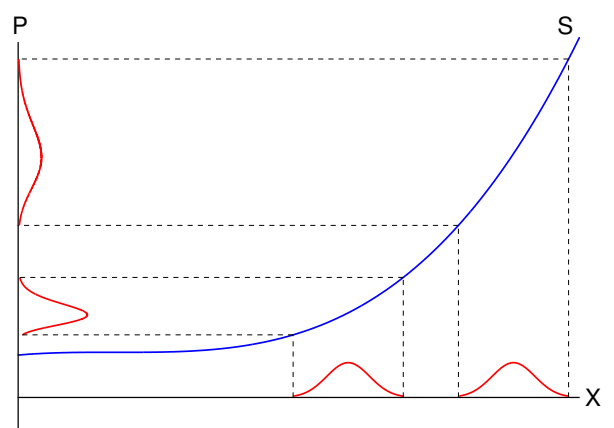

(a)

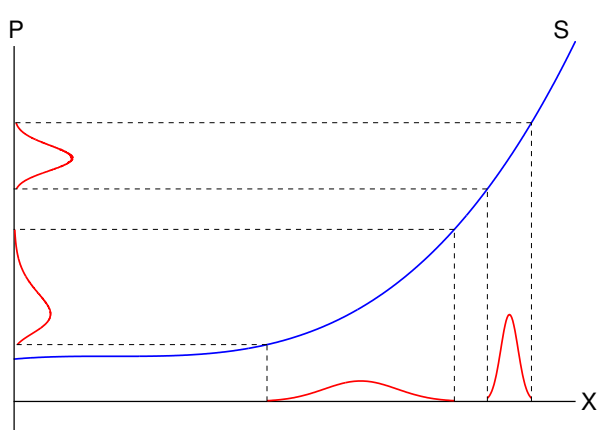

(b)

Figure 1: Panel (a): Shift of the residual demand with otherwise constant distribution. Panel (b): Shift of the residual demand with broadened distribution.

convex, and differentiable. We compare two scenarios with different shares of intermittent energy sources. In the first scenario the residual demand is given by $X$ with price $S(X)$. In the second scenario, we assume a larger share of IES and denote the additional random IES infeed by $I^{\prime} \geq 0$ and the resulting residual demand by $Y=X-I^{\prime}$. We start by investigating the case where the distribution of $Y$ is not broader than the distribution of $X$, which we formalize as

$$
F_{Y}^{-1}(\beta)-F_{Y}^{-1}(\alpha) \leq F_{X}^{-1}(\beta)-F_{X}^{-1}(\alpha), \quad \forall \alpha, \beta: 0 \leq \alpha<\beta \leq 1
$$

i.e., that the interquantile ranges of $X$ are as least as wide as that of $Y$. It is shown in Lemma 1 (see Appendix A) that (1) implies $\operatorname{Var}(Y) \leq \operatorname{Var}(X)$ (while the opposite is not necessarily true). We modify a theorem of See and Chen (2008) and obtain the following result, which we proof in the appendix.

Proposition 1. Let $X$ and $Y$ be non-negative random variables with inverse distribution functions $F_{X}^{-1}, F_{Y}^{-1}$ respectively, and let $S: \mathbb{R}^{+} \rightarrow \mathbb{R}$ be a convex increasing function which is twice differentiable. If (1) holds and $Y \leq X$, then

$$
\operatorname{Var}(S(Y)) \leq \operatorname{Var}(S(X))
$$

The proposition states under which conditions more IES leads to less price variance. Looking at the two conditions, we see that $Y \leq X$ holds by definition, but Condition (1) need not hold in practice. It is fulfilled for the special case depicted in Figure 1(a) where $Y=X-c$ for some constant $c>0$, but this does not hold in general. A thorough study of the proof of the above proposition shows that, depending on the interaction between the shape of the supply function and the distributions of the residual demand, the variance may still decrease, even if (1) is violated. However, this analysis involves tedious algebraic manipulation and leads to conditions that do not lend themselves to economic interpretation.

For a practical study, we disentangle the effect of the shape of the supply function and the distribution of residual demand on the price variance. We employ a first-order Taylor approximation of the supply function $S$ around $\mathbb{E}(X)=\mu$, which reads

$$
S(X) \approx S(\mu)+S^{\prime}(\mu)(X-\mu) .
$$




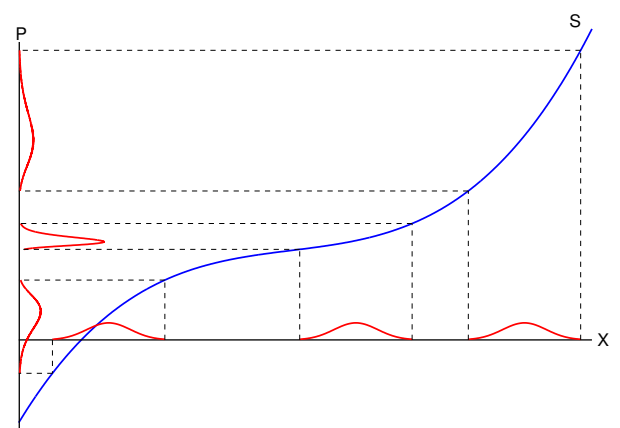

(a)

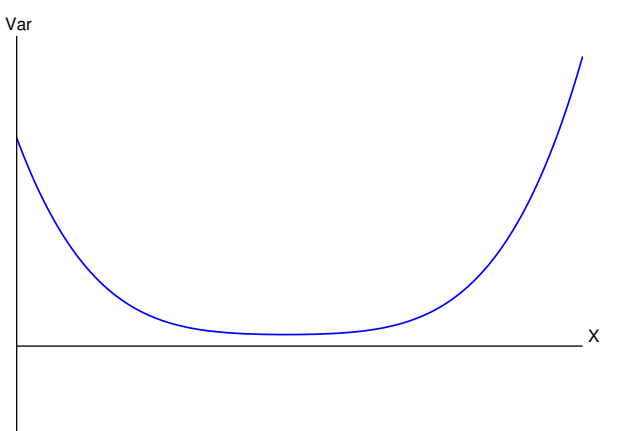

(b)

Figure 2: Panel (a): Price effects with a concave-convex supply function. Panel (b): Effects of a shift in the mean demand on the variance assuming a concave-convex supply function.

Taking variances on both sides using the fact that $\operatorname{Var}(a X+b)=a^{2} \operatorname{Var}(X)$ and $\operatorname{Var}(b)=0$ for $a, b \in \mathbb{R}$, we find

$$
\operatorname{Var}(S(X)) \approx S^{\prime}(\mu)^{2} \operatorname{Var}(X)
$$

Hence, the variance of the prices is approximated by the squared slope of $S$ evaluated at the mean of the residual demand times the variance of the residual demand. Analogously, the firstorder approximation for $\operatorname{Var}(S(Y))$ can be found and we see that $\operatorname{Var}(S(Y)) \leq \operatorname{Var}(S(X))$ is approximately true if

$$
S^{\prime}(\mathbb{E}(Y))^{2} \operatorname{Var}(Y) \leq S^{\prime}(\mathbb{E}(X))^{2} \operatorname{Var}(X)
$$

We can use the above criterion to explain what happens in Figure $1(\mathrm{~b})$ : From $Y \leq X$, it follows that $\mathbb{E}(Y) \leq \mathbb{E}(X)$ and therefore, since $S$ is convex, that $S^{\prime}(\mathbb{E}(Y)) \leq S^{\prime}(\mathbb{E}(X))$. Hence, to outweigh the effect that $Y$ is shifted to the flatter part of the supply curve, the variance of $Y$ has to be correspondingly bigger so that (5) is violated and the variance of $S(Y)$ is larger than the variance of $S(X)$. Conversely, if $Y$ is shifted far enough to the left, the convex shape of the supply function can outweigh a possible positive effect of $\operatorname{Var}(Y)$ on the price variance.

Condition (5) is no strict criterion, since it is based on an approximation which tends to get worse as the values of the random variables move away from their respective means. However, it formalizes the intuition underlying Figure 1.

Until now we have assumed a monotonically increasing, convex supply curve in a static model. In reality, the dispatch problem in one period is coupled with the dispatch decision in the neighboring periods, i.e., the dispatch is a dynamic decision. Nevertheless, the decisions of the players in the market result in an aggregate supply function for each hour, which can be used to study the effects of IES integration. Some authors argue that inflexible, slow base-load producers cause a dramatic decrease in prices near to the left end of the supply function. Such plants would rather accept producing at a loss for a few hours than switching off, which would induce longer down times and considerable fuel costs for restarting the plant (see e.g., Fanone et al, 2013). This corresponds to the observations made in Germany in times of low demand and high intermittent infeed, where prices frequently are close to zero and sometimes even drop to negative values. He et al (2013) adopt a dual-exponential model to capture this effect. In their paper, they show that a model with a supply curve consisting of a concave part followed by a convex part fits the merit order curve on the German spot market better than a purely convex supply function. We call a function of this type inverse $S$-shaped. This modification 
of the model thus incorporates the most important aspect of the dynamics of the dispatch decision, namely plant inflexibility and the resulting load changing costs.

The arguments in this section can be adapted to this setting as is demonstrated in Figure 2(a): If the demand is shifted into the concave part of the supply function then the variance tends to increase because of the steeper slope of the function in this region. Hence, in this setting, the variance as a function of the location of the mean of the residual demand, keeping all other characteristics of the distribution fixed, is U-shaped, i.e., high for very high and very low demand and smaller in the middle section as is depicted in Figure 2(b). In our empirical analysis, we show that the U-shaped dependence of the variance on the residual demand is present in German market data.

\section{Empirical analysis}

In Section 2, we identified the shape of the supply function and the distribution of the residual demand as the two pivotal influences on the electricity wholesale price variance. The findings imply that IES can either decrease or increase price variance, depending on which of the two influences prevail. In this section, we put these theoretical considerations to the test. For this purpose, we conduct a statistical investigation of the price variances currently observed in the German day-ahead spot market of electricity. More specifically, we set up OLS regression models, aiming to explain daily spot price variance by the variances and means of demand and renewable infeed, while controlling for other factors that also potentially affect electricity price variation. We are able to capture the variance increasing as well as the variance reducing effect in the regression model.

In this study, we define renewables as wind power and PV and do not consider other technologies. We do this for two reasons: First, because the bulk of intermittent production comes from these two technologies and second, because infeed from other relevant renewables, as for example biomass or hydro plants, can be controlled and therefore does not contribute much to the variance of the residual demand.

We start with an overview of the German market design with a focus on the day-ahead spot market and the marketing of renewable electricity and then discuss the variables we use in the regressions. In the main part of this section, we discuss the setup of the regressions as well as the results of our study of the price data from 2007 until 2013.

\subsection{The German Day-Ahead Market}

We pick Germany as a showcase because investment in renewable sources has been considerable in the last decade. Figure 3(a) documents this, showing the installed capacity of wind power and photovoltaics: installed capacity of wind plants was growing steadily between 2001 and 2012 with an average compound annual growth rate of about $12 \%$ and installed capacity of PV more than tripled between 2009 and 2012 (see also Wozabal et al, 2013).

For Germany the relevant market places to trade electricity are the European Energy Exchange (EEX) and the European Power Exchange (EPEX). While on the EEX options and futures on power can be traded, the EPEX hosts the day-ahead and intra-day market. The hourly prices on the day-ahead market are determined in sealed bid, uniform price auctions at 12 p.m. for every hour of the next day. On the intra-day market electricity can be traded continuously for delivery in single hours on the same or the following day until 75 minutes before delivery begins. 


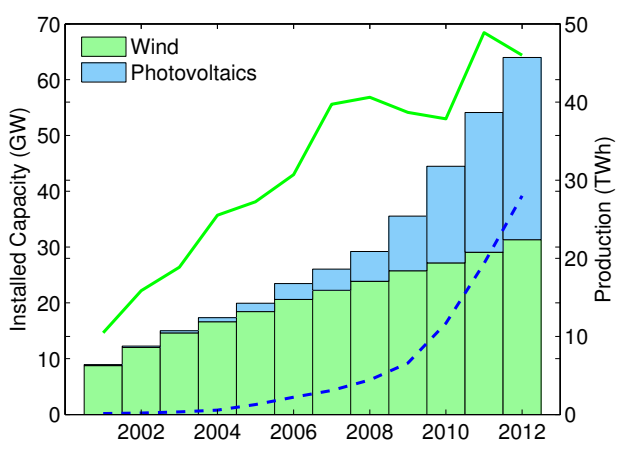

(a)

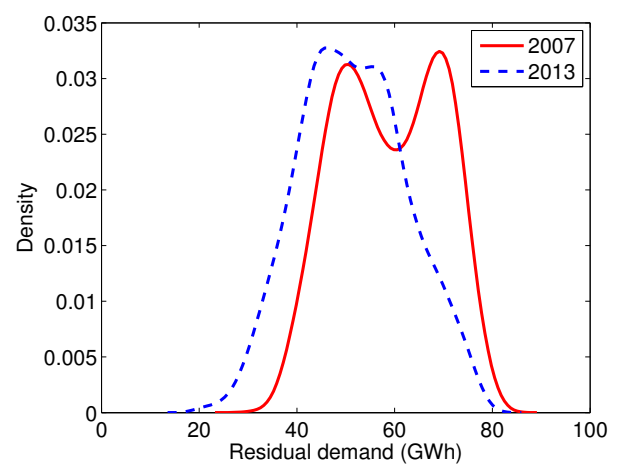

(b)

Figure 3: Panel (a): Installed capacity (bars) and production (lines) of wind power (solid) and PV (dotted) in Germany. Source: Wozabal et al (2013); Panel (b): Residual demand distributions (load reduced by forecast wind power and PV production).

The legal framework for subsidizing renewable power production in Germany is regulated in the German Renewable Energy Sources Act (see EEG, 2014) and its amendments, which requires the grid operators to buy all the power produced by renewable sources in their grid area for a regulated tariff and sell it further on the day-ahead and intra-day market. The grid operators have to publish forecasts of renewable production for the next day. The forecast quantities are sold on the day-ahead market and the differences between the day-ahead forecasts and actual volume are traded on the intra-day market.

The difference of the revenues on the spot markets and the payments to the producers of renewable energy are typically negative and are charged along with the grid fees to the final consumers. Hence, although producers receive a fixed feed-in tariff, renewable power is traded on the wholesale markets as if producers themselves would be active in these markets. In a recent modification of the EEG, producers of renewables can also opt for a market premium system instead of a feed-in tariff. In the market premium regime, producers sell their power directly on the market and receive a premium on top of the market price from the grid operators (see e.g., Gawel and Purkus, 2013). The modification of the EEG is in place since 2012 and aims to incentivize market-orientated behavior of the suppliers of renewables. Since most of the renewable production is sold on the day-ahead market, we focus on the day-ahead prices at the EPEX, where Germany and Austria are treated as one market zone. ${ }^{3}$

To guarantee transparency, the EEG requires grid operators to provide data on renewable production to the public. In particular, data about production and day-ahead forecasts of renewables is available in an 15 minutes resolution. The good data availability adds to the fact that the EPEX is an ideal showcase. Furthermore, Graf and Wozabal (2013) showed that the EPEX works efficiently, which means that the day-ahead market complies with the theoretical framework in Section 2.

\footnotetext{
${ }^{3}$ In principle, suppliers of renewables under the market premium regime are free in selecting a marketplace. However, the day-ahead spot market is way bigger than the intra-day market although the latter is growing: the traded volume in 2013 at the day-ahead market was 245.6 TWh (245.3 TWh in 2012) versus $19.7 \mathrm{TWh}$ on the intra-day market (15.8 TWh in 2012), see EPEX (2014). Therefore, we assume that most of the produced quantities are sold on the day-ahead market.
} 


\begin{tabular}{llll}
\hline Variable & Description & Source & Unit \\
\hline Price & Hourly day-ahead spot price for Germany/Austria & EPEX (2014) & EUR \\
Demand & Hourly load for Germany/Austria & ENTSO-E (2014) & GWh \\
Wind & Hourly day-ahead wind forecast for Germany/Austria & TSOs (2014) & GWh \\
Photovoltaic (PV) & Hourly day-ahead PV forecast for Germany & TSOs (2014) & GWh \\
Temperature & Daily temperature index & Mathematica & Celsius \\
Oil & Daily Europe Brent Spot Price FOB & EIA (2014) & EUR \\
Daylight minutes & Daily daylight minutes & & \\
\hline
\end{tabular}

Table 1: Overview of used variables and data sources.

\subsection{Data}

In this section, we discuss the variables used in our econometric analysis. An overview of these variables along with their descriptions and sources is provided in Table 1.

For our analysis, we use daily price variances, i.e., 24 hourly observations to compute one variance per day. The time frame to calculate the variance is chosen for the following reasons: On the one hand, it should be long enough such that the renewables can cause price variance, e.g., due to changed weather conditions. Thus, a higher resolution, e.g., half-daily, would be meaningless. On the other hand, the time frame should not be too long, since in this case one would loose important information due to aggregation and furthermore, we would end up with too few observations for a sound empirical analysis.

In order to estimate the effect of residual demand characteristics on the price variance, we require hourly data on prices, on electricity demand, and on infeed from renewable sources of power. The data on prices can be obtained directly from the EPEX website, the data on demand from the European Network of Transmission System Operators for Electricity (ENTSO-E) and the data on renewable infeed from the main grid operators (TSOs) in Germany/Austria. ${ }^{4}$

Considering the mechanisms of the day-ahead market, we use the day-ahead forecasts for renewable production instead of the actual infeeds, if available. For Germany, wind forecasts are available for the entire time period from 2007-2013. German PV data has not been published prior to $2010 .^{5}$ Austrian PV production is negligible and forecasts are not available, while Austrian wind production forecasts are published since 2010.

For the demand, day-ahead forecasts are not available in general, and we therefore substitute them by the actual load. The effect of this substitution should be marginal, since demand can be very well predicted. The mean average percentage error (MAPE) ${ }^{6}$ of the demand forecast is $2 \%$ for state of the art methods (see McSharry and Taylor, 2007; Taylor, 2010), as compared to the MAPE for wind power and PV production forecasts which range from $12 \%$ to $18 \%$, (Wozabal et al, 2013).

The characteristics of demand and infeed of renewable power are not the only factors driving price variance. To control for influences that originate from the supply side of the market, we include oil prices and three month lagged oil prices to capture the influence of the price of

\footnotetext{
${ }^{4}$ Germany: EnBW Transportnetze, Tennet TSO, Amprion, and 50Hertz Transmission; Austria: Austrian Power Grid.

${ }^{5}$ For 2010, only one of the german grid operators provided PV forecasts (Tennet TSO). Therefore, we extrapolated PV forecasts for the other grid operators by scaling up and down the Tennet forecasts according to the average ratios between the Tennet forecasts and the forecasts of the corresponding operator in 2011.

${ }^{6}$ The MAPE is defined as $n^{-1} \sum_{i=1}^{n}\left|\frac{y_{i}-\hat{y}_{i}}{y_{i}}\right|$ where $\left(\hat{y}_{i}\right)_{i=1}^{n}$ are the forecasts, $\left(y_{i}\right)_{i=1}^{n}$ are the actual values and $n$ is the total numbers of hours in the underlying period.
} 


\begin{tabular}{|c|c|c|c|c|c|c|c|c|}
\hline & 2007 & 2008 & 2009 & 2010 & 2011 & 2012 & 2013 & Total \\
\hline Price Avg & 35.85 & 64.83 & 38.93 & 44.51 & 51.50 & 42.51 & 37.60 & 45.11 \\
\hline Price Var & 430.04 & 672.55 & 252.16 & 174.68 & 158.01 & 202.90 & 235.11 & 391.62 \\
\hline Wind infeed Avg & 4.25 & 4.48 & 4.16 & 4.53 & 5.29 & 5.69 & 5.86 & 4.90 \\
\hline Wind infeed Var & 13.06 & 13.79 & 11.60 & 13.00 & 18.65 & 18.89 & 22.72 & 16.39 \\
\hline PV infeed Avg & & & & 1.11 & 2.27 & 3.18 & 3.40 & 2.49 \\
\hline PV infeed Var & & & & 3.25 & 11.08 & 22.86 & 27.93 & 17.11 \\
\hline Renewables infeed Avg & 4.25 & 4.48 & 4.16 & 5.64 & 7.56 & 8.87 & 9.27 & 6.32 \\
\hline Renewables infeed Var & 13.06 & 13.79 & 11.60 & 14.88 & 25.98 & 36.57 & 43.26 & 26.97 \\
\hline Demand Avg & 63.15 & 63.03 & 58.92 & 62.44 & 62.78 & 60.12 & 60.75 & 61.60 \\
\hline Demand Var & 117.50 & 109.15 & 122.93 & 109.44 & 125.34 & 132.11 & 121.96 & 122.14 \\
\hline Residual demand Avg & 58.90 & 58.55 & 54.75 & 56.80 & 55.22 & 51.26 & 51.49 & 55.28 \\
\hline Residual demand Var & 113.14 & 103.24 & 119.03 & 103.88 & 112.98 & 127.36 & 120.87 & 122.49 \\
\hline \# Observations & 8,729 & 8,753 & 8,730 & 8,760 & 8,759 & 8,784 & 8,760 & 61,275 \\
\hline \# Outliers $^{+}$ & 114 & 80 & 56 & 35 & 17 & 66 & 52 & 420 \\
\hline \# Outliers ${ }^{-}$ & 0 & 5 & 28 & 46 & 89 & 25 & 20 & 213 \\
\hline
\end{tabular}

Table 2: Descriptive statistics. Prices are reported in EUR/MWh while quantities are in GWh.

primary energy. ${ }^{7}$ Furthermore, we include the number of daylight minutes to capture seasonal trends, and an index of daily average temperatures. To construct the daily temperature index for Austria and Germany we use a population weighted mean of all the Austrian and German cities with more than 10,000 inhabitants. The index is constructed using the Mathematica 7.0 functions CityData and WeatherData (see Graf and Wozabal, 2013). For the oil price, we use the daily Europe Brent spot price provided by EIA (2014).

The reason we use the variance as the measure of dispersion is its nice theoretical properties. It allows for a neat decomposition of the residual demand in the regression models. This, in turn, permits to study in detail the two pivotal effects driving the price variance; other measures, e.g., standard deviation would not allow for such a decomposition. However, the variance is a quadratic expression and we are performing OLS regressions, minimizing the sum of squared residuals. The combination of the two renders our analysis extremely sensitive to outliers. Therefore, we remove hourly observations which deviate from the average price for every year by more than three standard deviations (see Mugele et al, 2005; Ketterer, 2012, for a similar approach). The corresponding numbers are shown at the bottom of Table $2 .{ }^{8}$ Moreover, we remove days where we have less than 13 price observations left, and we end up with 2553 daily observations (out of 2557 days in the considered time period).

\subsection{Descriptive Statistics}

Table 2 displays the descriptive statistics of our sample. Having a first glimpse on the raw data reveals several peculiarities: The yearly price variances of the years 2009 to 2013 are significantly lower than in the years 2007 and 2008, although the yearly average production

\footnotetext{
${ }^{7}$ Prices for primary energy are highly correlated. In particular, in the observation period, gas imported into continental Europe was mainly procured from long-term supply contracts. The price in these contracts is set with formulas which are based on the current oil price as well as the oil prices from three, six, or nine months (see Yegorov and Wirl, 2009). Hence, the lagged variable in the regression.

${ }^{8}$ For a few hours, data points are missing in the publicly available data, therefore the number of observations varies slightly over the years.
} 


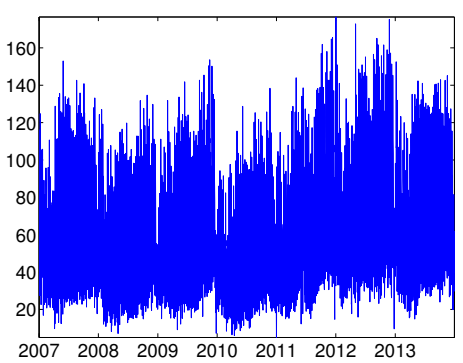

(a)

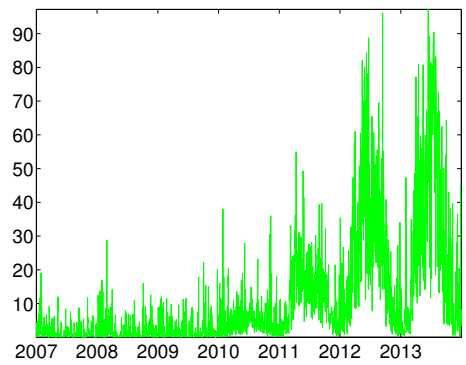

(b)

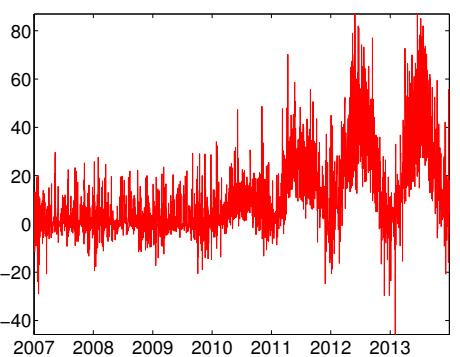

(c)

Figure 4: Panel (a): Variance of demand; Panel (b): Variance of renewable infeed; Panel (c): Covariance between demand and renewable infeed. Sample period 2007-2013. Demand and renewable infeed in GWh.

of renewables increased drastically in the same time. The yearly empirical variance of the renewables has increased with the massive build-up of photovoltaics $(2011-2013)$. Wind production obviously exhibits long-term variability, since e.g., average yearly production is lower in 2009 than in 2007 although installed capacity is significantly higher in 2009. We also note that the number of negative outliers in the price peaks in 2011 while the number of positive outliers decreased from 2007 to 2011.

While the average renewable production increased considerably between 2007 and 2013, the yearly average demand decreased slightly, leading to a changed residual demand. Figure 3(b) shows the results of a kernel density estimation of residual demand for the first and the last year in our sample. Comparing the two years yields the following observations: (i) the mean decreased between 2007 and 2013, (ii) the shape of the distribution changed, in particular the two peaks are less pronounced in 2013 than in 2007, (iii) the maximum residual demand stayed almost constant while the minimum residual demand reduced drastically. The changing shapes of the distributions can be explained by PV production - a technology which supplies most electricity during the midday peak hours. Therefore, an increase in PV production eliminates the traditional noon peak which has an effect on the shape of the residual demand distribution (see e.g., Gawel and Purkus, 2013; Tveten et al, 2013).

When studying the influence of IES on the variance of residual demand, the covariances between demand and renewable infeed obviously play an important role too. In Figure 4, we depict the daily development of the variances of demand and renewables as well as their covariances. While the daily demand variance changes only slightly between the years (Panel a), the introduction of PV in 2010 has a significant impact on the intra-day variance of renewable infeed (Panel b). PV also affected the covariance between demand and renewables (Panel c), since this technology supplies the most power during the noon hours, a time of the day which usually coincides with high demand.

\subsection{Results}

To test the influence of residual demand, wind power, and PV infeed on the variance of German wholesale electricity prices, we estimate the following OLS regression models with the price variance as dependent variable: A model that uses residual demand as an explanatory variable (model 1), a model in which the components that make up residual demand, i.e., demand and 
IES infeed, are treated as separate regressors (model 2), and a third model where we further disentangle the infeed from renewable sources into their two constituting elements, i.e., wind power and PV (model 3).

All models include the other factors discussed in the last section in daily resolution. We perform all regressions using heteroskedasticity and autocorrelation robust standard errors (Andrews, 1991; Zeileis, 2006), since both the Durbin-Watson test and the Breusch-Pagan test reject the hypothesis of i.i.d. residuals for our models. For the statistical analysis we used the program $R$ (R Core Team, 2013).

We first discuss the results from model 1, where we use the distribution of the residual demand to explain price variance. In particular, we are interested in the effects of shifts in the residual demand and in changes in the shape of the distribution. In the regressions, we capture the former by average daily residual demand and model the latter by daily variances of demand. To include the possibility of an inverse S-shaped supply function, we also include the squared average daily demand as a regressor. It turns out that both the AIC and the BIC favor the corresponding model over the model without the squared term.

The results of the regression are depicted in Table 3 in the column marked with (1). The average residual demand has a convex quadratic influence on the price variance, which leads to higher variances for very low and very high levels of residual demand. This can be seen as an indication of an inverse S-shaped supply function. The variance of the residual demand has a strong positive influence on the variance of the prices. Both empirical findings support the theoretical considerations in Section 2, which state that changes in the price variance are driven by shifts into areas with different slope of the supply curve and by changes in the dispersion of the residual demand distribution.

To capture the influence of renewables on the variance, we estimate model 2 , i.e., we break up the residual demand and repeat the analysis. Motivated by the results about the quadratic relationship of the price variance on the average residual demand, we also include all the terms of the expansion

$$
X^{2}=(Q-I)^{2}=Q^{2}-2 Q I+I^{2},
$$

i.e., the squared demand, the squared amount of intermittent production, and the interaction of the two. Following a similar logic, we split up the variance of the residual demand as

$$
\operatorname{Var}(X)=\operatorname{Var}(Q-I)=\operatorname{Var}(Q)-2 \operatorname{Cov}(Q, I)+\operatorname{Var}(I)
$$

and include all the terms on the right hand side of the above equation as regressors.

Table 3, Column 2, shows the results of the regression for model 2. The coefficients of the covariance between demand and renewables and the interaction term of these two variables have the anticipated negative signs. Figure 5(a) illustrates how the predicted variance is influenced by the average amount of renewable production. The results suggest that a higher demand generally leads to a higher price variance than a lower demand, except if the low demand occurs in combination with high infeed from renewables. The co-occurrence of low demand and high infeed of renewables pushes the residual demand into the steep area of the inverse S-shaped supply curve. This results in extremely low prices and a higher price variance and relates to the phenomenon of negative prices for electricity on the day-ahead spot market that are sometimes observed in the German market (see e.g., Fanone et al, 2013). One reason for this are inflexible base load plants which, to avoid start-up cost, prefer to produce at negative prices instead of switching off.

With regard to the variance of demand and renewables, the coefficients of model 2 show that the price variance increases with an increase in the variance of demand and renewables while the covariance between those has a negative effect. Figure $5(\mathrm{~b})$ depicts these effects. The upper 


\begin{tabular}{|c|c|c|c|}
\hline & (1) & $(2)$ & $(3)$ \\
\hline Intercept & $\begin{array}{l}981.73 \\
(0.001)\end{array}$ & $\begin{array}{l}152.55 \\
(0.729)\end{array}$ & $\begin{array}{r}4.67 \\
(0.990)\end{array}$ \\
\hline Residual Demand & $\begin{array}{l}-40.06 \\
(0.000)\end{array}$ & & \\
\hline Residual Demand ${ }^{2}$ & $\begin{array}{r}0.43 \\
(0.000)\end{array}$ & & \\
\hline Demand & & $\begin{array}{l}-15.59 \\
(0.256)\end{array}$ & $\begin{array}{l}-16.19 \\
(0.186)\end{array}$ \\
\hline Demand $^{2}$ & & $\begin{array}{r}0.23 \\
(0.0067)\end{array}$ & $\begin{array}{r}0.23 \\
(0.036)\end{array}$ \\
\hline Renewables & & $\begin{array}{r}18.64 \\
(0.096)\end{array}$ & \\
\hline Renewables $^{2}$ & & $\begin{array}{r}1.07 \\
(0.000)\end{array}$ & \\
\hline Renewables * Demand & & $\begin{array}{c}-0.69 \\
(0.002)\end{array}$ & \\
\hline Wind & & & $\begin{array}{r}29.74 \\
(0.011)\end{array}$ \\
\hline Wind $^{2}$ & & & $\begin{array}{r}0.50 \\
(0.007)\end{array}$ \\
\hline PV & & & $\begin{array}{l}-53.71 \\
(0.023)\end{array}$ \\
\hline $\mathrm{PV}^{2}$ & & & $\begin{array}{r}9.93 \\
(0.000)\end{array}$ \\
\hline Wind ${ }^{*}$ Demand & & & $\begin{array}{l}-0.74 \\
(0.001)\end{array}$ \\
\hline $\mathrm{PV} *$ Demand & & & $\begin{array}{l}-1.13 \\
(0.023)\end{array}$ \\
\hline $\mathrm{PV} *$ Wind & & & $\begin{array}{r}2.52 \\
(0.004)\end{array}$ \\
\hline Residual Demand Var & $\begin{array}{r}1.70 \\
(0.000)\end{array}$ & & \\
\hline Demand Var & & $\begin{array}{r}1.15 \\
(0.000)\end{array}$ & $\begin{array}{r}1.02 \\
(0.000)\end{array}$ \\
\hline Renewables Var & & $\begin{array}{r}1.88 \\
(0.000)\end{array}$ & $\begin{array}{r}2.75 \\
(0.000)\end{array}$ \\
\hline Demand, Renewables Cov & & $\begin{array}{r}-5.92 \\
(0.000)\end{array}$ & $\begin{array}{r}-3.59 \\
(0.000)\end{array}$ \\
\hline Daylight Hours & $\begin{array}{r}-0.06 \\
(0.510)\end{array}$ & $\begin{array}{r}0.03 \\
(0.720)\end{array}$ & $\begin{array}{r}0.11 \\
(0.222)\end{array}$ \\
\hline Temperature & $\begin{array}{r}3.76 \\
(0.019)\end{array}$ & $\begin{array}{r}4.42 \\
(0.004)\end{array}$ & $\begin{array}{r}3.95 \\
(0.004)\end{array}$ \\
\hline Oil Price & $\begin{array}{r}-2.66 \\
(0.136)\end{array}$ & $\begin{array}{l}-2.34 \\
(0.122)\end{array}$ & $\begin{array}{r}-1.07 \\
(0.420)\end{array}$ \\
\hline Oil Price 3M & $\begin{array}{r}2.71 \\
(0.126)\end{array}$ & $\begin{array}{r}4.05 \\
(0.012)\end{array}$ & $\begin{array}{r}5.21 \\
(0.000)\end{array}$ \\
\hline $\begin{array}{l}\text { Adjusted } R^{2} \\
N\end{array}$ & $\begin{array}{r}0.25 \\
2,553\end{array}$ & $\begin{array}{r}0.29 \\
2,553\end{array}$ & $\begin{array}{r}0.36 \\
2,553\end{array}$ \\
\hline
\end{tabular}

Table 3: Coefficients (p-values below) of the OLS regressions for the years 2007 to 2013 (heteroskedasticity and autocorrelation consistent estimation of the variance-covariance matrix). All variables represent daily averages unless indicated otherwise.

plane shows the situation for the lowest observed covariance between demand and renewable 
infeed in our data while the lower plane represents the situation with the highest observed covariance. The interpretation of this effect is the following: an increased variance of demand and renewables leads to a higher price variance. However, this effect is less pronounced for a high covariance between these two variables than for a low covariance. This is in particular interesting because Figure 4(c) shows fluctuating level of covariance over the years.

Finally, we discuss the results of model 3, where we split up renewable production into its parts, i.e., wind power and PV. However, due to the almost perfect correlation (0.95) between the variance of $\mathrm{PV}$ and its mean, we do not include both terms in the regression. The high correlation is caused by the peculiarity of PV, i.e., it supplies only during daylight hours and a higher variance is observed when more electricity from PV is supplied. Thus, a full decomposition into the sample mean and variance of wind and PV is not possible. Instead, we only decompose the mean of the renewable production. We present the results in Table 3, Column 3. The effects of the average wind and PV production on the relative price variance are depicted in Figure 6. Notice that the relative price variance is evaluated at the average demand of $61.6 \mathrm{GW}$ (see Table 2). The estimated coefficients of PV infeed and the coefficient of the interaction effect between PV and demand are reflected in the sharp decrease of price variance when average $\mathrm{PV}$ infeed increases. Wind infeed also reduces the price variance, but not as drastically. Although both coefficients of wind infeed are positive, the price variance is reduced because of the negative interaction term between wind infeed and demand. The average marginal effects of PV and wind infeed (per GWh) on the price variance evaluated at the observations yield an average reduction of 83 for PV compared to 7 in the case of wind. Therefore, the impact of average PV infeed on the price variance is about twelve times larger than that of wind.

The coefficients of the variables which are not related to the residual demand are of similar magnitude in all three models: Price variance is increasing with average temperature, leading to higher price variance in summer than in winter. ${ }^{9}$ As expected, the average three month lagged oil price which serves as a proxy for the fossil fuel prices has a positive influence on the price variance. The coefficients of the average daylight minutes and the oil price are not significant. The explained variation ranges from 25 to 36 percent.

As an additional exercise, we performed yearly regressions in order to identify anomalies between the different years. With one exception all relevant coefficients have the same sign as in the full sample. The results of these regressions are available on request.

\section{Policy Implications}

In this paper, we argue that the effect of IES infeed on the spot price variance depends crucially on the amount and the type of installed capacity. First, the higher the correlation of the infeed with stochastic demand the higher the variance dampening effect brought about by a decrease of the variance of residual demand. In this sense, the installation of PV capacities tends to decrease price variance more than the installation of wind turbines, since the infeed from the former has a pronounced positive correlation with demand, while the infeed from the latter shows no such clear correlation pattern. Political assessments of the desirability of wind power versus PV should take this aspect into account.

Second, according to our analysis in markets with inverse S-shaped supply functions the introduction of a small to moderate amount of IES is likely to have a variance damping effect

\footnotetext{
${ }^{9}$ Since Germany and Austria are traditionally winter-peaking countries this seems counterintuitive at first glance. An explanation is that high PV production in summer coupled with lower demand pushes the residual demand in the concave area of the merit order resulting in a higher variance.
} 


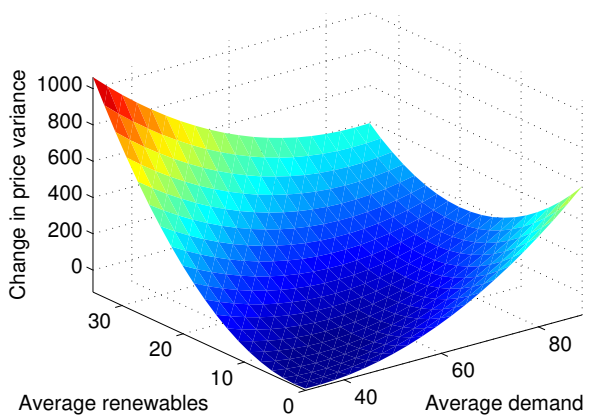

(a)

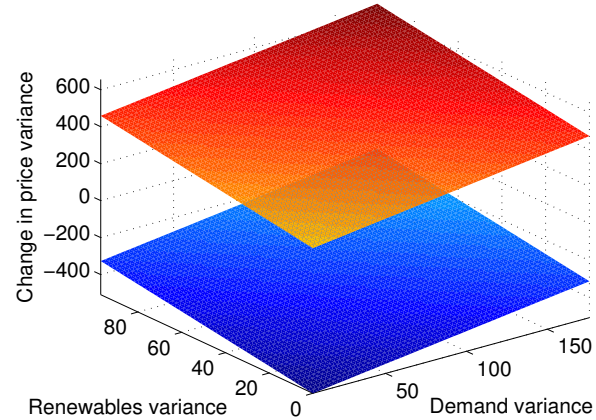

(b)

Figure 5: Panel (a): Predicted effects of average renewable infeed and demand (in GWh) on relative price variance; Panel (b): Change in price variance in dependence on renewable infeed and demand variance at the two extreme levels of observed covariance. All other regressors are set to zero.

while a larger increase may lead to an increased price variance on the electricity exchanges, as the residual demand is pushed into the concave part of the merit order.

In fact, we can quantify the tipping point for the German market using the models' estimates from Section 3. Given the estimated coefficients of model 2, we can find the daily demand and the renewable infeed that minimize the predicted variance in prices. Carrying out this exercise yields a demand of $42.8 \mathrm{GWh}$ and a renewables infeed of about 5.7 GWh. Fixing the demand at its average of about $61.6 \mathrm{GWh}$, the minimal variance is reached at a level of renewable infeed of $12 \mathrm{GWh}$. Notice that these numbers are daily mean and variance levels, i.e., the daily price variance is minimized given a daily infeed of renewables and a fixed demand. Although these calculations give only a rough picture, it becomes clear that in the first years of our analysis, the IES infeed will have had a variance dampening effect on most days, while in the later years, with increased capacity and a large number of high infeed days, IES will have increased the variance on many days.

For policy makers this has important implications. Clearly, the structural changes in electricity markets, triggered by the build-up of intermittent renewable electricity production, require increased flexibility of production and consumption. This flexibility may either be provided by electricity storage plants, by a demand response via for example smart grids, or by flexible production technologies such as gas turbines (see Denholm and Hand, 2011).

Policies to promote renewable electricity production typically directly target the building of corresponding production capacities, tacitly assuming that the demand for increased flexibility will result in market conditions that act as incentives to invest in demand side response technologies and storage facilities. However, all corresponding technologies require substantial investments whose returns are mainly driven by price variance. In particular, if price variance goes down in the first phase of renewable expansion, then these projects become less profitable and correspondingly less capacities will be build or projects will be delayed, even if firms correctly anticipate an increase in price variance at a later point in time.

Since construction of the corresponding infrastructure takes time, this in turn might result in a situation where the overall system is left with too little flexibility to handle increased IES infeed which may lead to network problems and, in the worst case, blackouts. Examples of near 


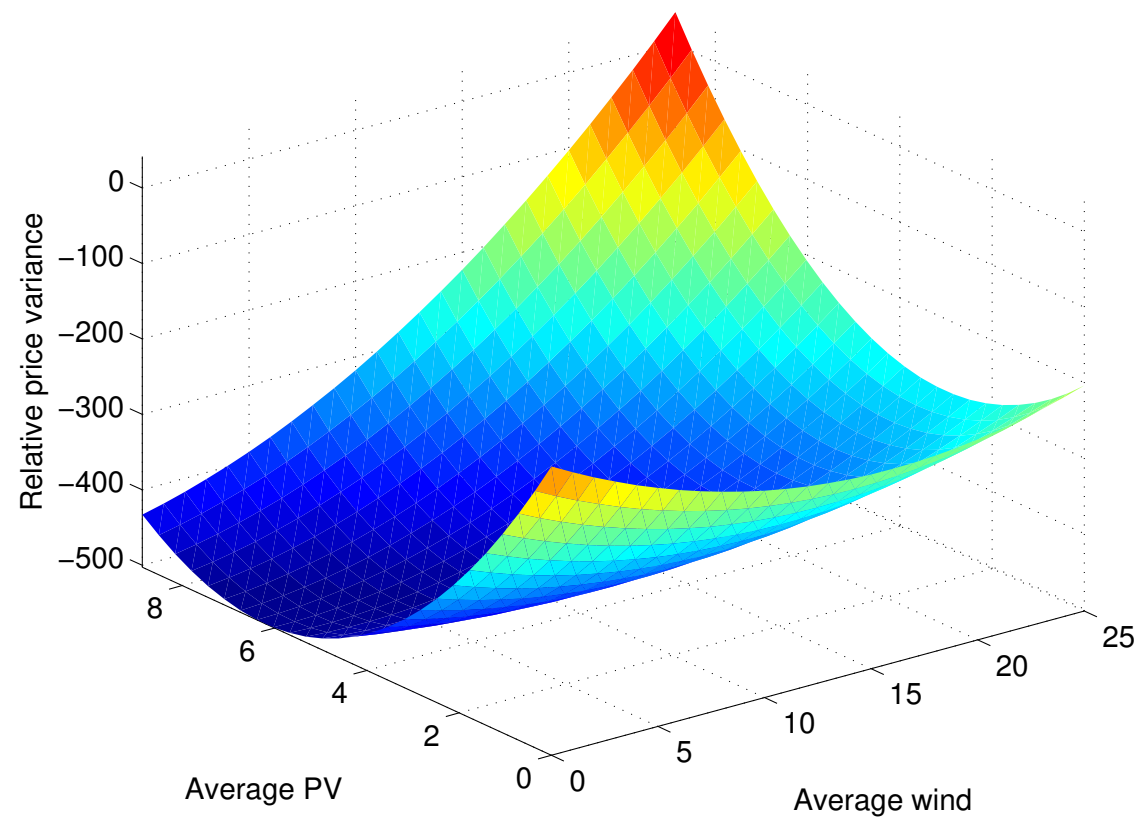

Figure 6: Predicted effects of average PV and wind infeed (in GWh) on relative price variance evaluated at the average demand level of $62.6 \mathrm{GWh}$. All other regressors are set to zero.

grid failures in Germany could be observed in the year 2011 (see Bundesnetzagentur, 2012). Since there is no market for the economic costs of blackouts these costs constitute a negative externality which might decrease overall welfare, even if all players act rational on the existing electricity markets.

In conclusion, it seems reasonable to complement policies that subsidize the build-up of renewable intermittent capacities with policies or market designs that incentivize the expansion of storage capacity, some means of demand response, or transmission capacities to neighboring grid areas. A large scale demand response by consumers could for example be facilitated by a smart electricity grid in combinations with flexible pricing schemes for electric power, (see e.g., Strbac, 2008). Flexibility in the production and storage capacity can either be directly subsidized or ensured by capacity markets (see e.g., Crampton et al, 2013, for a discussion on the importance of capacity markets). The promotion of electric cars whose combined battery capacity can act as a storage device might be another option if combined with a smart grid (see e.g., Guille and Gross, 2009).

Which of these measures is best suited to address the problem is subject to further research and might also depend on the specifics of the market in question.

\section{Conclusion}

While the short-term negative effect of renewables on average spot prices is beyond controversy in the literature, research on price variance is still rather scarce and most authors conclude that variance increases with the installation of intermittent capacities. In this paper we showed that the build-up of IES can not only increase but also decrease electricity price variance. The latter 
effect is expected to occur for rather small amounts of IES capacity, while the former is expected once a significant amount of IES capacity is installed. We argue this point theoretically using a static market model and identify the slope of the supply function as well as the variance of the IES infeed as two crucial factors affecting price variance. Whereas the variance of IES infeed increases price variance, the actual amount of IES infeed can also influence the price variance negatively. In Section 3, we test our theoretical considerations and quantify the two effects using data from the German spot market. As we argue in the last section, market based incentives for investments in flexibility alone might not result in sufficient capacities built in time to ensure network stability. Policies that require producers to provide flexibility, the build-up of infrastructure such as a smart grid, capacity markets, or direct subsidies for flexible generation and storage facilities might mitigate these potential market failures. Which of these alternatives is the most efficient is an interesting question for further research.

Further investigations about the price variance might want to explicitly incorporate the dynamics in the bidding that determines the market prices, for example in a dispatch model. Another direction of future research could be the modelling of strategic interactions of the players on the market, an aspect which we did not consider here.

\section{References}

Andrews DWK (1991) Heteroskedasticity and Autocorrelation Consistent Covariance Matrix Estimation. Econometrica 59:817-858

Arocena P, Saal DS, Coelli T (2012) Vertical and Horizontal Scope Economies in the Regulated U.S. Electric Power Industry. The Journal of Industrial Economics 60(3):434-467

Barlow M (2002) A diffusion model for electricity prices. Mathematical Finance 12(4):287-298, DOI 10.1111/j.1467-9965.2002.tb00125.x

Bundesnetzagentur (2012) Report on Energy Supplies in Winter 2011/12. Tech. rep.

Chao H (2011) Efficient pricing and investment in electricity markets with intermittent resources. Energy Policy 39(7):3945-3953, DOI 10.1016/j.enpol.2011.01.010

Crampton P, Ockenfels A, Stoft S (2013) Capacity Market Fundamentals. Economics of Energy \& Environmental Policy 2(2):27-46

Denholm P, Hand M (2011) Grid flexibility and storage required to achieve very high penetration of variable renewable electricity. Energy Policy 39(3):1817-1830

Dunne T, Mu X (2010) Investment spikes and uncertainty in the petroleum refining industry. The Journal of Industrial Economics 58(1):190-213

EEG (2014) Renewable Energy Sources Act (EEG). Online, available: http://www.erneuerbare-energien.de

EIA (2014) U. S. Energy Information Administration. Online, available: http://www.eia.gov

ENTSO-E (2014) European Network of Transmission System Operators for Electricity. Online, available: http://www.entsoe.eu, http://www.entsoe.net

EPEX (2014) European Power Exchange. Online, available: http://www.epexspot.com 
Fanone E, Gamba A, Prokopczuk M (2013) The case of negative day-ahead electricity prices. Energy Economics 35:22-34

Gawel E, Purkus A (2013) Promoting the market and system integration of renewable energies through premium schemes - A case study of the German market premium. Energy Policy 61:599-609

Graf C, Wozabal D (2013) Measuring competitiveness of the EPEX spot market for electricity. Energy Policy 62:948-958

Green R, Vasilakos N (2010) Market behaviour with large amounts of intermittent generation. Energy Policy 38(7):3211-3220, DOI 10.1016/j.enpol.2009.07.038

Guille C, Gross G (2009) A conceptual framework for the vehicle-to-grid (v2g) implementation. Energy Policy 37:4379-4390

Gurland J (1967) An inequality satisfied by the expectation of the reciprocal of a random variable. The American Statistician 21:24-25

He Y, Hildmann M, Herog F, Andersson G (2013) Modeling the merit order curve of the european energy exchange power market in germany. IEEE Transactions on Power Systems $28(3): 3155-3164$

Hirth L (2013) The market value of variable renewables: The effect of solar wind power variability on their relative price. Energy Economics 38:218-236

Jacobsen HK, Zvingilaite E (2010) Reducing the market impact of large shares of intermittent energy in Denmark. Energy Policy 38(7):3403-3413, DOI 10.1016/j.enpol.2010.02.014

Jónsson T, Pinson P, Madsen H (2010) On the market impact of wind energy forecasts. Energy Economics 32:313-320, DOI 10.1016/j.eneco.2009.10.018

Ketterer JC (2012) The Impact of Wind Power Generation on the Electricity Price in Germany. Ifo Working Paper Series 143, Ifo Institute for Economic Research at the University of Munich

Kim DW, Knittel CR (2006) Biases in static oligopoly models? Evidence from the California electricity market. The Journal of Industrial Economics 54(4):451-470

McSharry P, Taylor J (2007) Short-term load forecasting methods: an evaluation based on European data. IEEE Transaction on Power Systems 22(4):2213-2219

de Miera GS, del Ro Gonzlez P, Vizcano I (2008) Analysing the impact of renewable electricity support schemes on power prices: The case of wind electricity in Spain. Energy Policy $36(9): 3345-3359$

Milstein I, Tishler A (2011) Intermittently renewable energy, optimal capacity mix and prices in a deregulated electricity market. Energy Policy 39(7):3922-3927, DOI 10.1016/j.enpol. 2010.11.008

Muche T (2009) A real option-based simulation model to evaluate investments in pump storage plants. Energy Policy 37(11):4851-4862

Mugele C, Rachev ST, Trück S (2005) Stable modeling of different european power markets. Investment Management and Financial Innovations 3:65-85 
Paraschiv F, Erni D, Pietsch R (2014) The impact of renewable energies on eex day-ahead electricity prices. Energy Policy (Article in Press)

R Core Team (2013) R: A Language and Environment for Statistical Computing. R Foundation for Statistical Computing, Vienna, Austria, URL http://www.R-project.org/

Redl C, Haas R, Huber C, Böhm B (2009) Price formation in electricity forward markets and the relevance of systematic forecast errors. Energy Economics 31(3):356-364

See CT, Chen J (2008) Inequalities on the variances of convex functions of random variables. Journal of inequalities in pure and applied mathematics 9:Article 80

Strbac G (2008) Demand side management: Benefits and challenges. Energy Policy 36:44194426

Taylor JW (2010) Triple seasonal methods for short-term electricity demand forecasting. European Journal of Operational Research 204(1):139 - 152

TSOs (2014) German TSOs: Amprion, 50 Hertz, TenneT, and TransnetBW; Austrian TSO: APG. Online, available: http://www.amprion.net, www.50hertz.com, http://www.tennettso.de, www.transnetbw.de, www.apg.at

Tveten Å, BolkesjøTF, Martinsen T, Hvarnes H (2013) Solar feed-in tariffs and the merit order effect: A study of the German electricity market. Energy Policy 61:761-770

Wagner A (2014) Residual Demand Modeling and Application to Electricity Pricing. The Energy Journal 35(2):45-73

Woo C, Horowitz I, Moore J, Pacheco A (2011) The impact of wind generation on the electricity spot-market price level and variance: The texas experience. Energy Policy 39(7):3939-3944, DOI 10.1016/j.enpol.2011.03.084

Wozabal D, Graf C, Hirschmann D (2013) Renewable Energy and its Impact on Power Markets. In: Kovacevic, Pflug, Vespucci (eds) Handbook of Risk Management for Energy Production and Trading, International Series in Operations Research and Management Science, Springer, pp 283-311

Yegorov Y, Wirl F (2009) Ukrainian gas transit game. Zeitschrift fr Energiewirtschaft 33(2):147-155

Zeileis A (2006) Object-oriented Computation of Sandwich Estimators. Journal of Statistical Software 16(9):1-16 


\section{A Appendix}

The proof of Proposition 1 requires the following proposition:

Proposition 2. Let $X$ be a positive random variable which takes values on an interval $[a, b]$. Let $h: \mathbb{R}^{+} \times \mathbb{R} \rightarrow \mathbb{R}$ be a function such that the following hold:

1. $h(X, \cdot)$ is twice differentiable

2. $h(\cdot, \epsilon)$ is increasing $\forall \epsilon \in[a, b]$, and

3. $\frac{\partial}{\partial \epsilon} h(\cdot, \epsilon)$ is decreasing $\forall \epsilon \in[a, b]$.

Then for $\epsilon_{1}, \epsilon_{2} \in[a, b]$ with $\epsilon_{1}<\epsilon_{2}$ it holds true that $\operatorname{Var}\left[h\left(X, \epsilon_{2}\right)\right] \leq \operatorname{Var}\left[h\left(X, \epsilon_{1}\right)\right]$.

Proof. The proof is analogue to the proof of (See and Chen, 2008, Theorem 3.1). It suffices to show that $\operatorname{Var}[h(X, \epsilon)]$ is a decreasing function of $\epsilon$. We have

$$
\begin{aligned}
\frac{\partial}{\partial \epsilon} \operatorname{Var}(h(X, \epsilon)) & =\frac{\partial}{\partial \epsilon} \mathbb{E}\left(h(X, \epsilon)^{2}\right)-\frac{\partial}{\partial \epsilon}\left(\mathbb{E}(h(X, \epsilon))^{2}\right. \\
& =\mathbb{E}\left[\frac{\partial}{\partial \epsilon} h(X, \epsilon)^{2}\right]-2 \mathbb{E}(h(X, \epsilon)) \frac{\partial}{\partial \epsilon}(\mathbb{E}(h(X, \epsilon)) \\
& =\mathbb{E}\left[2 h(X, \epsilon) \frac{\partial}{\partial \epsilon} h(X, \epsilon)\right]-2 \mathbb{E}(h(X, \epsilon))\left(\mathbb{E}\left(\frac{\partial}{\partial \epsilon} h(X, \epsilon)\right)\right. \\
& \leq 0 .
\end{aligned}
$$

The last inequality follows from the fact that for a random variable $X$, and for continuous functions $f, g$ on $\mathbb{R}, \mathbb{E}[f(X) g(X)] \leq \mathbb{E}[f(X)] \mathbb{E}[g(X)]$ if $f$ is monotonically increasing and $g$ is monotonically decreasing (see e.g., Gurland (1967)). The interchange of the differential operator and the expectation is clearly justified by the assumptions we have made.

Proof of Proposition 1. The proof is analogue to (See and Chen, 2008, Theorem 3.3). We sketch the important steps.

Define $h(u, \epsilon)=g\left(F_{X}^{-1}(u)+\epsilon\left[F_{Y}^{-1}(u)-F_{X}^{-1}(u)\right]\right)$, with $\epsilon \in[0,1]$. First, note that $h(\cdot, \epsilon)$ is an increasing function $\forall \epsilon \in[0,1]$. Secondly, note that $\frac{\partial}{\partial \epsilon} h(\cdot, \epsilon)$ is an decreasing function since

$$
\begin{aligned}
\frac{\partial}{\partial u} \frac{\partial}{\partial \epsilon} h(\cdot, \epsilon)= & \frac{\partial}{\partial u}\left\{\left[F_{Y}^{-1}(u)-F_{X}^{-1}(u)\right] g^{\prime}\left(F_{X}^{-1}(u)+\epsilon\left[F_{Y}^{-1}(u)-F_{X}^{-1}(u)\right]\right)\right\} \\
= & {\left[\left(F_{Y}^{-1}\right)^{\prime}-\left(F_{X}^{-1}\right)^{\prime}\right] g^{\prime}\left(F_{X}^{-1}(u)+\epsilon\left[F_{Y}^{-1}(u)-F_{X}^{-1}(u)\right]\right) } \\
& +\epsilon\left(F_{X}^{-1}\right)^{\prime}\left[F_{Y}^{-1}-F_{X}^{-1}\right] g^{\prime \prime}\left(F_{X}^{-1}(u)+\epsilon\left[F_{Y}^{-1}(u)-F_{X}^{-1}(u)\right]\right) \\
& +(1-\epsilon)\left(F_{X}^{-1}\right)^{\prime}\left[F_{Y}^{-1}-F_{X}^{-1}\right] g^{\prime \prime}\left(F_{X}^{-1}(u)+\epsilon\left[F_{Y}^{-1}(u)-F_{X}^{-1}(u)\right]\right) \\
\leq & 0
\end{aligned}
$$

For the last inequality note that the first term is negative due to condition (2) and $g$ being increasing $\left(g^{\prime}>0\right)$, while the second and third term are negative due to condition (1), convexity of $g\left(g^{\prime \prime}>0\right)$ and properties of distribution functions, $\left(F_{X}^{-1}\right)^{\prime} \geq 0$ and $\left(F_{Y}^{-1}\right)^{\prime} \geq 0$.

Now one can apply Proposition 2 and finds

$$
\operatorname{Var}[g(Y)]=\operatorname{Var}[h(u, 1)] \leq \operatorname{Var}[h(u, 0)]=\operatorname{Var}[g(X)] .
$$


The following lemma links inequality (1) to the variance of the random variables.

Lemma 1. If for two random variables $X$ and $Y$

$$
F_{Y}^{-1}(\alpha)-F_{X}^{-1}(\alpha)
$$

is monotonically decreasing, then $\operatorname{Var}(Y) \leq \operatorname{Var}(X)$.

Proof. Notice that for $\alpha>\alpha^{\prime}$, we have

$$
F_{Y}^{-1}(\alpha)-F_{X}^{-1}(\alpha) \leq F_{Y}^{-1}\left(\alpha^{\prime}\right)-F_{X}^{-1}\left(\alpha^{\prime}\right)
$$

and therefore

$$
F_{Y}^{-1}(\alpha)-F_{Y}^{-1}\left(\alpha^{\prime}\right) \leq F_{X}^{-1}(\alpha)-F_{X}^{-1}\left(\alpha^{\prime}\right),
$$

i.e., the inter-quantile range of $Y$ is smaller than that of $X$ for all quantiles $\alpha, \alpha^{\prime}$.

Setting $\alpha_{1}=F_{X}(\mathbb{E}(X))$ and $\alpha_{2}=F_{Y}(\mathbb{E}(Y))$, yields

$$
\begin{aligned}
\operatorname{Var}(\mathrm{X}) & =\int_{-\infty}^{\infty}(x-\mathbb{E}(X))^{2} f_{X}(x) d x=\int_{0}^{1}\left(F_{X}^{-1}(\alpha)-F_{X}^{-1}\left(\alpha_{1}\right)\right)^{2} d u \\
& \geq \int_{0}^{1}\left(F_{Y}^{-1}(\alpha)-F_{Y}^{-1}\left(\alpha_{1}\right)\right)^{2} d u \geq \int_{0}^{1}\left(F_{Y}^{-1}(\alpha)-F_{Y}^{-1}\left(\alpha_{2}\right)\right)^{2} d u \\
& =\operatorname{Var}(Y),
\end{aligned}
$$

where the last inequality follows from

$$
\mathbb{E}(Y)=F_{Y}^{-1}\left(\alpha_{2}\right)=\arg \min \left\{a: \mathbb{E}\left[(Y-a)^{2}\right]\right\} .
$$

